Trauma Berufskrankh 2004 · 6 [Suppl 4] : S424-S432 DOI 10.1007/s10039-004-0894-5

Online publiziert: 20. Mai 2004

(c) Springer-Verlag 2004

R. Feil ${ }^{1}$ A. Rübberdt ${ }^{1,2} \cdot$ A. Ekkernkamp ${ }^{1}$

${ }^{1} K$ Klinik für Unfall- und Wiederherstellungschirurgie, Unfallkrankenhaus, Berlin

2Unfall- und Wiederherstellungschirurgie, Charité Universitätsmedizin, Campus Benjamin Franklin, Berlin

\title{
Aktueller Stand der Behandlungsmöglichkeiten von Knorpeldefekten
}

\section{Knorpel-Knochen- vs. autologe Chondrozytentransplantation}

Entnahme- und Transplantationstechnik in Sinne einer OATS-Plastik aus dem biologischen Aspekt überlegen scheint.

Der Eindruck, dass wirtschaftliche Ursachen die Indikation beeinflussen, kann letztendlich nicht von der Hand gewiesen werden, da es sich um ausgesprochen kostenaufwändige Verfahren und Operationsinstrumentarien handelt. Derartige Einflüsse sollten das Handeln des Therapeuten keinesfalls, insbesondere innerhalb von Studien, beeinflussen.

Eine belastbare Datenbasis bezüglich der Art des Knorpeldefekts und des indizierten Ersatzverfahrens ist aufgrund der bisher veröffentlichten Untersuchungen nicht zu verzeichnen.

\section{Erarbeitung von Richtlinien}

Auch wenn keine ausreichende Datenlage zur weiteren Auswertung zur Verfügung steht, sollten auf der Grundlage zellbiologischer Erkenntnisse sowie der klinischen Erfahrung von Zentren, welche die rekonstruktive Gelenkchirurgie intensiviert betreiben, Richtlinien erarbeitet werden, anhand derer die Indikationen der einzelnen Verfahren der Knorpelrekonstruktion klar aufgezeigt werden.
Die einzelnen Verfahren haben in den Untersuchungen ihre Wirksamkeit nachweisen können [14], wobei die ACT der herkömmlichen OATS-Plastik überlegen scheint [8].

Das Diamant-Nass-Schleif-Verfahren überzeugt durch Präzision und gute Transplantationstechnik mittels Pressfit. Die Technik erfuhr durch die Auffüllung der Hebedefekte durch Zylinder vom Beckenkamm einen deutlichen Fortschritt $[5,7]$.

Auch die ACT verzeichnete Verbesserungen auf dem Gebiet der Operationstechnik $[6,16]$.

\section{Behandlung \\ isolierter Knorpelveränderungen des Kniegelenks}

Im Unfallkrankenhaus Berlin werden folgende Zuordnungen getroffen.

ACT

Grundlegend ist zu bemerken, dass auch sie ein Verfahren darstellt, welches eine Früharthrose vermeiden soll, aber nicht zu den Eingriffen zählt, die ihren Platz in der Arthrosebehandlung suchen. 


\section{Indikationen}

Die korrekte Indikationsstellung ist von entscheidender Bedeutung für den Erfolg der Behandlung mittels autologer Chondrozytentransplantation. Sowohl die Erkenntnisse aus dem Labor als auch klinische Beobachtungen zeigten, dass die Zellen, welche für die extrakorporale Vermehrung gewonnen werden, einen möglichst hochwertigen Zustand aufweisen müssen, um ein entsprechendes Regenerat erwarten zu können. Arthrotische oder anderweitig geschädigte Zellen weisen ein differentes Proliferationsverhalten mit erhöhter Apoptoserate auf [12]. Hierdurch werden die ersten Rahmenbedingungen für die Indikationsstellung festgelegt.

Die Transplantation von autologen Chondrozyten eröffnet die Möglichkeit einer Rekonstruktion eines isolierten Knorpelschadens Grad III-IV nach Outerbridge in einem Ausmaß von $2-10 \mathrm{~cm}^{2}[3$, 14, 16]. Verletzungen des Knorpels, welche eine intakte Knochenstruktur erkennen lassen, können mittels dieser Methode behandelt werden $[1,3,14,16]$. Eine Veränderung der grundplattennahen Knochenstruktur sollte nicht vorliegen, dann ist eine optimale Ernährung der Knorpelzellen auch von Seiten des Knochens her anzunehmen.

Es ist nicht letztendlich geklärt, inwieweit die Ernährung der Chondrozyten von Seiten der Synovialflüssigkeit im Verhältnis zur Knochengrundplatte erfolgt. Eine ausgeprägte Veränderung der Knochenstruktur wie bei der Osteochondrosis dissecans lässt hier eine suboptimale Situation bezüglich der Versorgung des Knorpelgewebes erwarten.

Die in - Abb. 1 dargestellte Situation stellt die ideale Indikation zur Durchführung einer Transplantation von körpereigenen Chondrozyten nach extrakorporaler Vermehrung dar: Es liegt ein Knorpeldefekt vor, wobei die Knochensubstanz keine wesentlichen strukturellen Veränderungen aufweist.

Die Refixation eines Flakes, wie er in - Abb. 2 dargestellt ist, verläuft frustran, da der Knorpel nicht in der Lage ist, eine mechanisch stabile Verbindung zum Knochen auszubilden.

Trauma Berufskrankh 2004 · 6 [Suppl 4] : S424-S432

DOI 10.1007/s10039-004-0894-5

(c) Springer-Verlag 2004

R. Feil · A. Rübberdt · A. Ekkernkamp

Aktueller Stand der Behandlungsmöglichkeiten von Knorpeldefekten. Knorpel-Knochen- vs. autologe Chondrozytentransplantation

\section{Zusammenfassung}

Auch ohne Kenntnis der zellbiologischen Grundlagen ist schon seit Jahrhunderten bekannt, dass Veränderungen des Knorpels - sowohl degenerativer als auch traumatischer Art - einer Behandlung nur ausgesprochen schwer zugänglich sind. Da weder operative noch konservative Methoden eine Regeneration von originärem Gelenkknorpel herbeiführen können, zielten die Maßnahmen auf eine Entlastung des geschädigten Gewebes oder die Proliferation von Ersatzgewebe im Defektareal ab. In keinem Fall konnte eine Restitutio ad integrum erreicht werden. Basierend auf den Erfolgen der zellbiologischen Grundlagenforschung der letzten Zeit zeigten Studien, die sich mit der Transplantation von extrakorporal vermehrten Knorpelzellen befass- ten, sehr gute Erfolge. Die Transplantation von originärem Gelenkknorpel im Verbund mit Knochengewebe erfuhr durch Innovationen auf dem Gebiet der Operationsinstrumente ebenfalls erhebliche Fortschritte. Die höchste Präzision bei der Fertigung von Schleifen mit Diamantbeschichtung ermöglicht inzwischen eine Technik, welche mittels Pressfit-Verpflanzung eine Defektdeckung ohne verbleibende Zwischenräume erlaubt. Eine klare Indikationstrennung der einzelnen Verfahren existiert derzeit weder in der Literatur noch im klinischen Alltag.

\section{Schlüsselwörter}

Knorpel · Chondrozyten · Arthrose .

Osteochondrale Läsion · Transplantation

\section{Current status of treatment options for cartilaginous defects. Transplantation of cartilage-bone aggregates vs autologous chondrocytes}

\section{Abstract}

Even centuries ago, before much was known about the cell biology of cartilage, it was clear that trauma and degenerative disease affecting cartilage were not easily amenable to treatment. As neither operative nor conservative methods have so far been able to initiate the regeneration of cartilage, the methods used have been aimed at reducing mechanical stress on the damaged tissue or stimulating the overgrowth of defects by fibrous tissue. These efforts have never resulted in the restoration of a normal joint surface. Based on recent successful research in cell biology, many studies have now documented considerable progress in the transplantation of chondrocytes grown in vitro. Another boost for the trans- plantation of joint cartilage together with bony tissue came with the development of new surgical instruments. In particular, the extremely high-precision methods used in production of the new diamond-coated cutting instruments has made it possible to apply a press-fit technique allowing really accurate defect coverage. Nonetheless, the literature does not contain any classification of the different techniques by indication; nor is any such classification reflected in clinical practice.

\section{Keywords}

Cartilage · Chondrocytes · Degenerative Joint Disease · Osteochondral lesion · Transplantation 


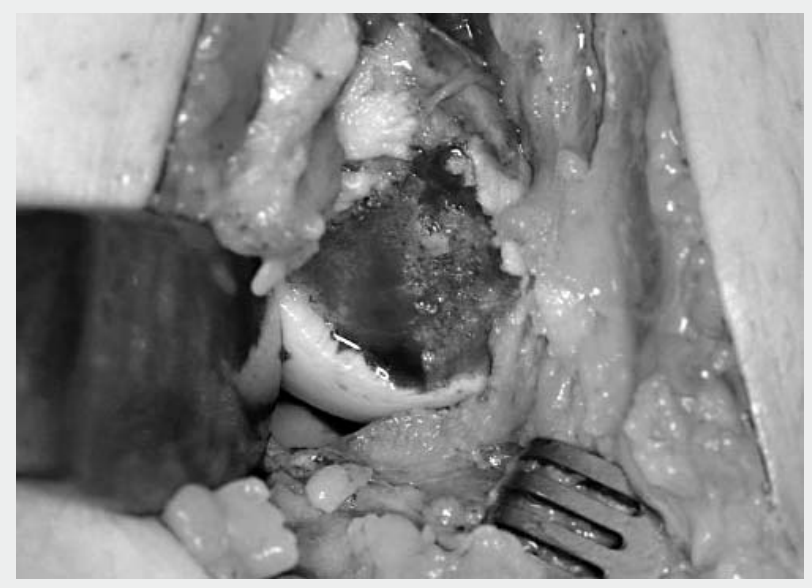

Abb. $1<$ Knorpelschaden Grad 4 des Femurkondylus

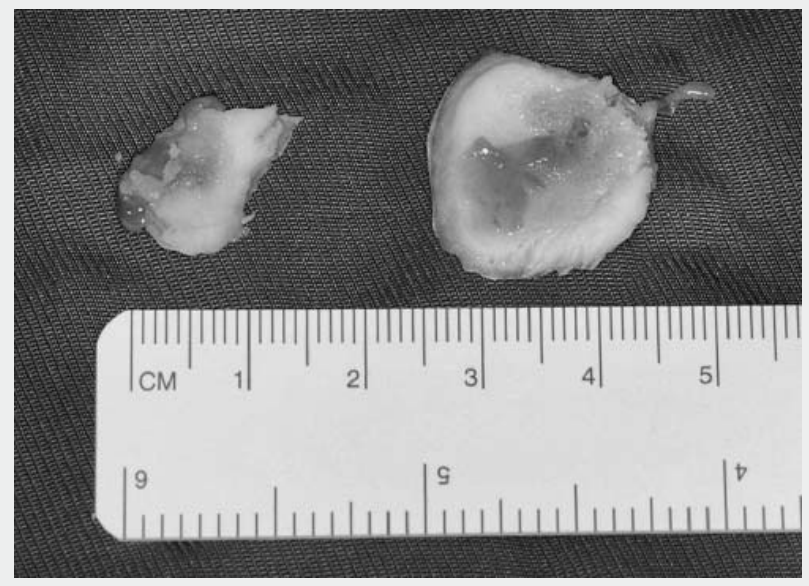

Abb. $2<$ Knorpelflake

\section{Kontraindikationen}

Hier sind zu nennen [16]:

- tief greifende Defekte mit Knochenbeteiligung

- Osteonekrosen

- Gelenkinfektionen

- rheumatoide Arthritis

- Pangonarthrosen

- gegenüberliegende korrespondierende Defekte

- ligamentäre Instabilität

- Inkongruenz der Gelenk bildenden ossären Strukturen

- neoplastische Grunderkrankungen

- biologisches Alter >50 Jahren

Ligamentäre Instabilitäten müssen zuvor einer stabilisierenden Versorgung zugeführt werden, wie z. B. einer Ersatzplastik des vorderen Kreuzbands. Bei entsprechenden Fehlstellungen sind Umstellungsoperationen erforderlich $[10,14,16]$.

\section{Operationstechnik}

Die autologe Chondrozytentransplantation ist kein Verfahren, welches in einem Arbeitsgang durchgeführt werden kann. Vorausgehen müssen die eingehende Anamneseerhebung, körperliche Untersuchung und eine Magnetresonanztomographie, wodurch das Ausmaß des Defekts dreidimensional beurteilt werden kann. Primär werden nach Indikationsstellung im Rahmen einer Arthroskopie das gesamte Kniegelenk sowie die Defektzone inspiziert und die Indikation somit erneut überprüft.

Nach Ausschluss der Kontraindikationen erfolgt die Entnahme eines Knorpelchips außerhalb des Defektbezirks, welcher qualitativ hochwertig erscheint. In der Regel liegt die Entnahmestelle nahe der Notch, ohne dass der Hauptbelastungsbezirk tangiert wird (• Abb. 3). Das arthroskopisch entnommene Präparat wird umgehend in einem sterilen Transportmedium weitergeleitet. Ein serologischer Test auf HIV-1/-2, HBV und HCV ist obligat. Willigt ein Patient hierzu nicht ein, ist die Herstellung eines Chondrozytenregenerats auch aus forensischer Sicht nicht möglich.

Der entnommene Knorpel wird im Zelllabor einer mechanischen und enzymatischen Behandlung unterzogen. Die dadurch aus ihrer Matrix herausgelösten Knorpelzellen werden in vitro kultiviert.

Nach Abschluss der Vermehrungsphase, welche etwa 2-4 Wochen in Anspruch nimmt, werden die Zellen dem Operationsteam zur Verfügung gestellt. Mittels einer Arthrotomie erhält man $\mathrm{Zu}$ gang zur Defektzone des Knorpels. Der restlich bestehende Knorpel der geschädigten Zone wird entfernt, die subchondrale Grenzzone bleibt unversehrt, um die Migration von Fibroblasten zu vermeiden (• Abb. 4).

Ein Periostlappen vom Tibiakopf oder dem Femurkondylus wird entnommen, um die Defektzone so zu decken, dass eine Kammer entsteht. Hierzu wird der Lappen mittels feiner Nähte am gesunden Knorpel fixiert und die Übergangszone mit Fibrinkleber abgedichtet. Ist die Kammer dicht, wird die Knorpelzellsuspension in sie hinein injiziert. Nach Verschluss der Wunde ist die Operation abgeschlossen.

Der in - Abb. 4 dargestellte Eingriff zeigt die Verwendung einer weiteren Entwicklung auf dem Sektor der ACT. Anstelle eines Periostlappens wird eine Kollagenmembran verwendet. Die mit ihr erzielten Ergebnisse scheinen derzeit die

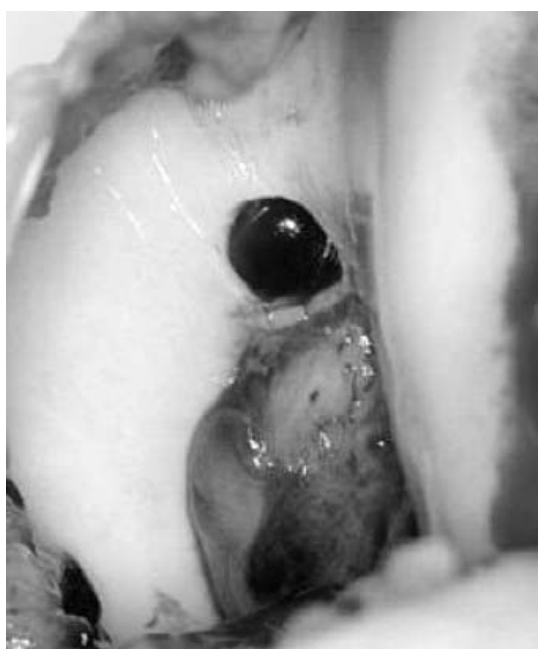

Abb. $3 \Delta$ Entnahmestelle Knorpelgewebe, Entnahme nach erfolgter Arthrotomie 
Resultate der Periostlappentechnik zu übertreffen, da die Membran nicht, wie der Periostlappen, Mediatoren enthält, welche eine mögliche Hypertrophie des Regenerats begünstigen können. Auch wird eine Vermischung der Gewebestrukturen unterbunden.

Eine eingenähte Kollagenmembran wird innerhalb von etwa 3-4 Monaten resorbiert.

Auch Defekte des Knorpels der Kniescheibe sind der Therapie zugänglich (• Abb. 5 a-d).

\section{Nachbehandlung}

Sie erfordert ein differenziertes Konzept, welches sich an der Lokalisation des Defekts und der angewandten Operationsmethode orientiert. In der Regel ist eine Abrollbelastung der betroffenen Extremität für etwa 6 Wochen erforderlich. Nach einer Ruhigstellung bis zur Entfernung der Redon-Dränagen kann die frühfunktionelle Übungsbehandlung mittels Motorschiene, Bewegungsübungen und Isometrie begonnen werden. Nach 3 Monaten ist eine sportliche Betätigung im Sinne von Radfahren und Schwimmen möglich, nach 6 Monaten auch Joggen, und nach 1 Jahr können auch Betätigungen wie Skifahren ausgeübt werden.

Die in $\bullet$ Abb. 6 gezeigte Kontrollarthroskopie 6 Monate nach Durchführung einer ACT zeigt ein mechanisch vollwertiges Regenerat über dem medialen $\mathrm{Fe}$ murkondylus im Hauptbelastungsbereich. Die Transplantation erfolgte in unserer Klinik unter Verwendung einer Kollagenmembran. Die Chondrozyten wurden in serenfreien Medien gezüchtet.

\section{Knorpel-Knochen-Transplantation}

\section{Indikationen}

Bei Verletzungen oder Erkrankungen der subchondralen Knochensubstanz ist eine Transplantation von Knorpel-KnochenZylindern im Diamant-Nass-Schleif-Verfahren zu bevorzugen.

Diamantbeschichtete Schleifen eignen sich zur Entnahme und Transplantation von Knorpel- und Knochengewebe. Die Wandstärke der Fräse beträgt o,4 mm. Bei dieser Technik kann ein Substanzdefekt nahezu vermieden werden. Die Diamantbeschichtung trennt die spongiöse Struk-
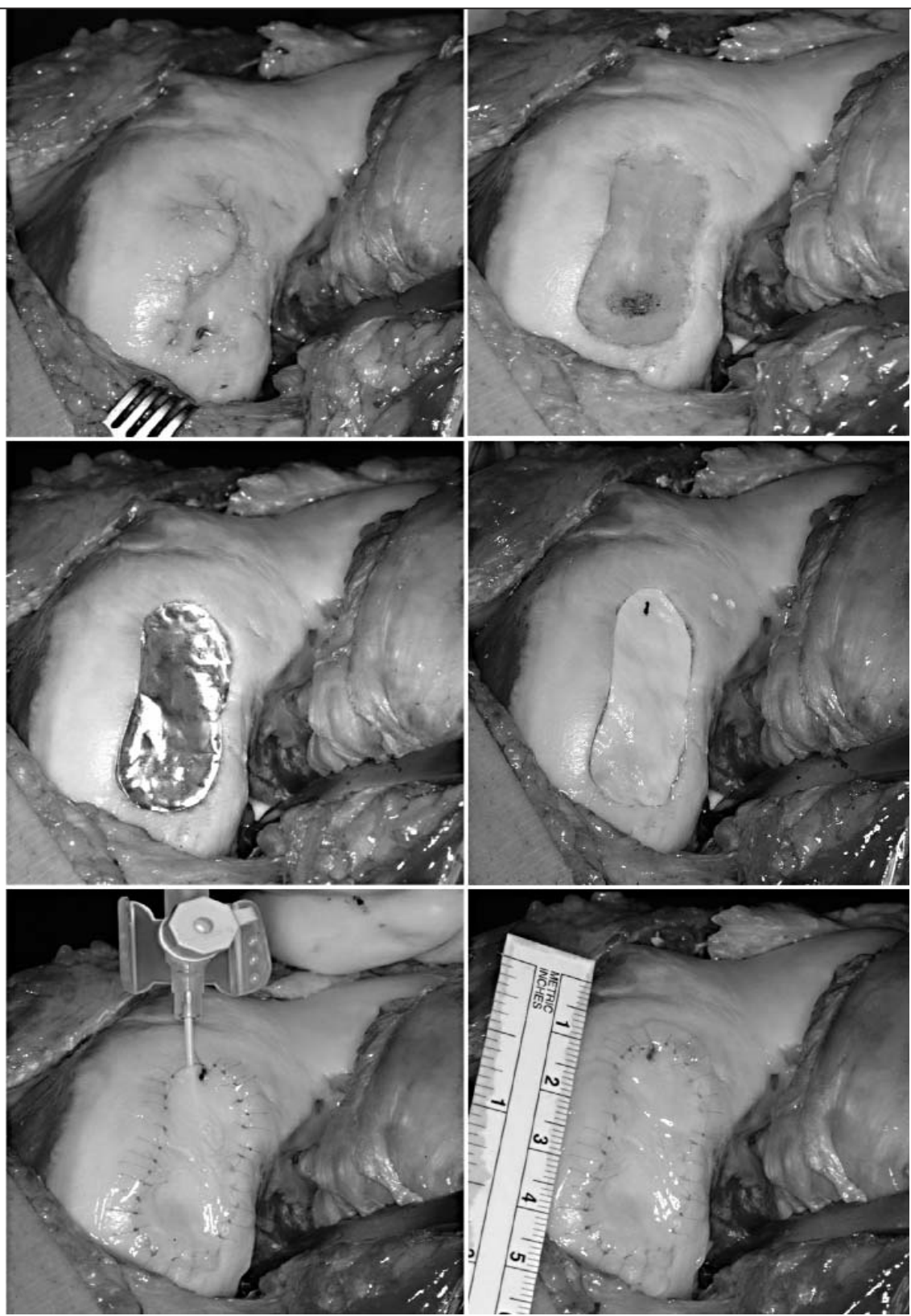

Abb. $4 \Delta$ Operative Versorgung mittels ACT

tur des Entnahmezylinders aus seiner Umgebung, ohne die originäre Knochenbälkchenform zu brechen, wie es bei herkömmlichen Entnahmetechniken die Regel ist. Eine feine Größenabstufung der Fräsen und maximale Präzision im Fertigungsgang lassen eine bisher unerreichte Passgenauigkeit des Zylinders in seinem neu geschaffenen Lager erzielen (• Abb. 7).

Wesentliche Merkmale der Transplantate, welche mittels der Surgical-DiamondInstruments (SDI) entnommen und in Pressfit-Technik verpflanzt wurden, sind:
- eine hohe Stabilität im Sinne einer primären Belastbarkeit

- eine rapide Revaskularisierung

- eine rasche knöcherne Konsolidierung

Die Hebedefekte werden ebenfalls in Pressfit-Technik mit periostbedeckten Zylindern aufgefüllt, welche aus dem Beckenkamm entnommen werden. Das Periost besitzt die Fähigkeit, binnen etwa 6-8 Wochen zu Faserknorpel zu differenzieren. Somit kann eine für die im femoropatellaren Lager bestehende mecha- 

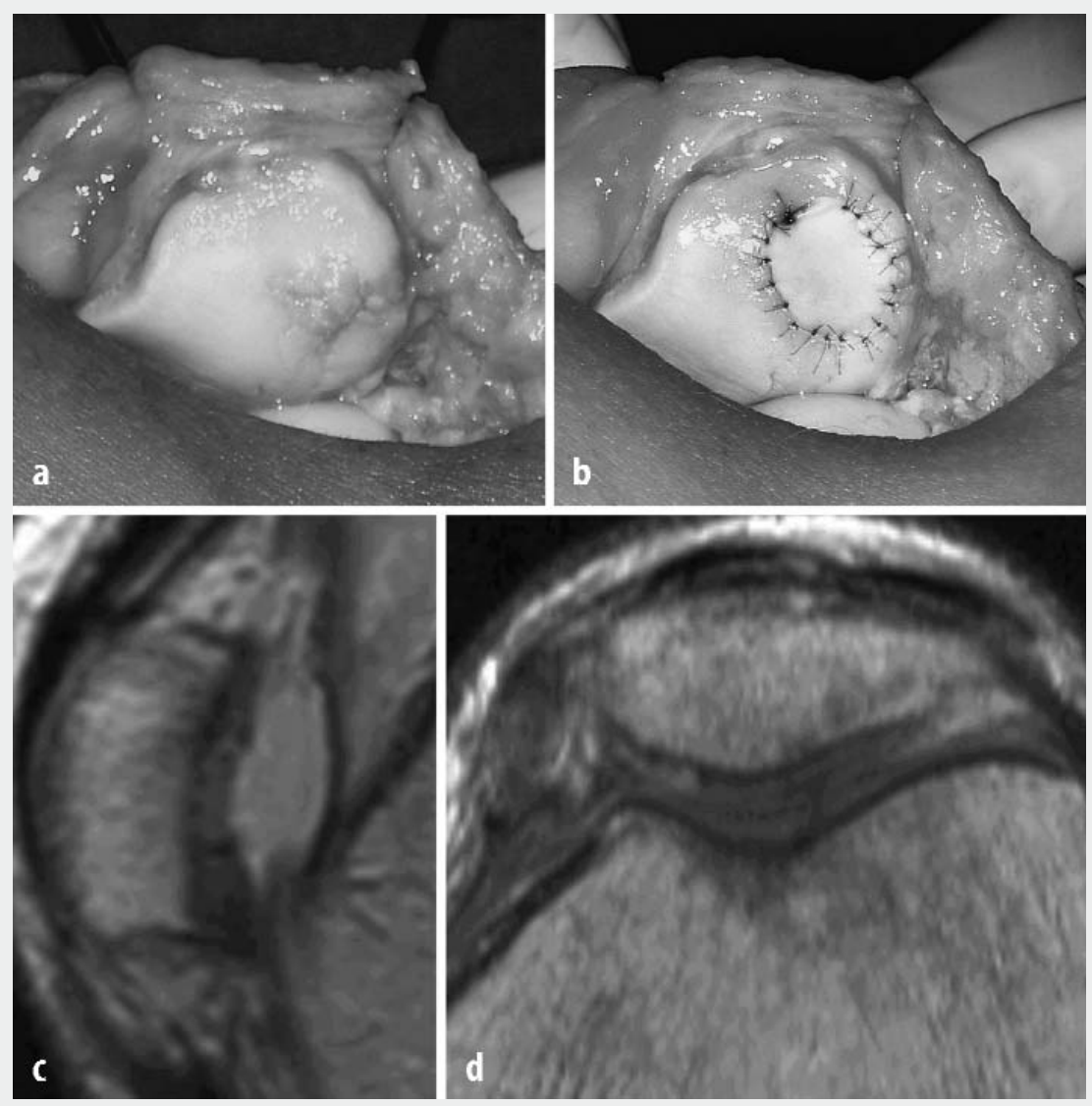

Abb. 5a-d $\Delta$ Retropatellarer Defekt (a), eingenähte Membran (b), Kontroll-MRT 6 Monate postoperativ, gute Auffüllung des Defekts mit Regeneratknorpel ( $c, d)$

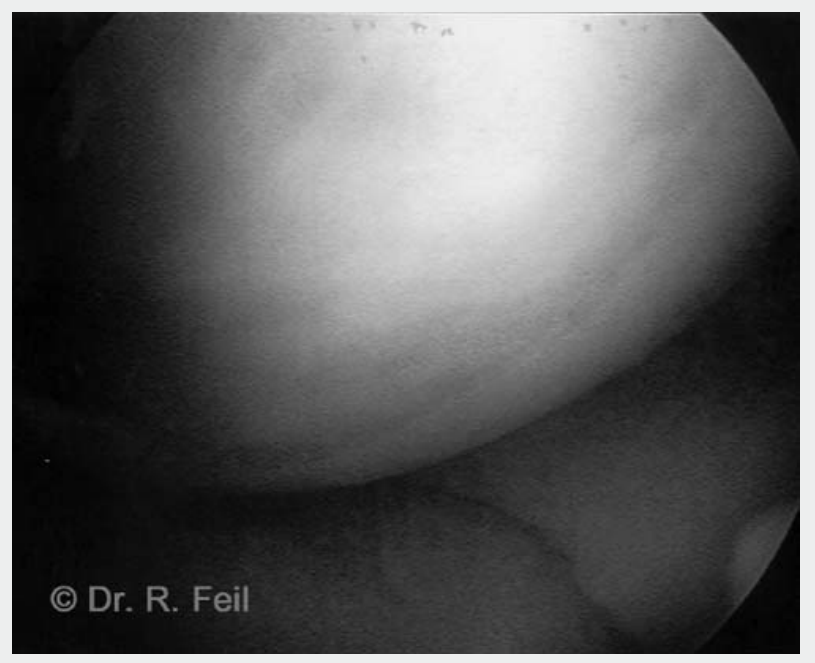

Abb. $6<$ Kontrollarthroskopie 6 Monate nach ACT

nische Beanspruchung suffiziente Knorpeldeckung erzielt werden.

Die Möglichkeit der überlappenden Anordnung mehrerer Zylinder in PressfitTechnik lässt erstmalig eine Defektdeckung von größeren Arealen zu, welche eine bisher unerreichte Belastbarkeit bei Verwendung von biologisch aktiven und vitalen
Transplantaten ermöglicht (• Abb. 8). Zwischenräume innerhalb des transplantierten Areals mit Ausbildung eines minderwertigen Regenerats, wie sie bei den herkömmlichen Techniken regelmäßig zu beobachten waren, können vollständig vermieden werden $[5,7,11]$. Die in $\bullet A b b .9$ gezeigte Magnetresonanztomographie
6 Wochen nach einer gleichartigen Operation zeigt die exakte Passgenauigkeit der transplantierten Zylinder mit Rekonstruktion der Gelenkfläche.

Insbesondere die Osteochondrosis dissecans des Kniegelenks oder des Talus bietet sich als Indikation für eine KnorpelKnochen-Transplantation an. Von wesentlicher Bedeutung ist die Nekrose des Knochens, welche die Nutrition des Knorpels über die Knochengrundplatte unterbindet und den Untergang der Chondrozyten bedingen kann. Auf dem Grund der Nekrose ist ein Angehen einer vitalen Zellkultur ohne Schäden als nicht wahrscheinlich anzunehmen. Somit ist eine Wiederherstellung der intakten Stoffwechselsituation mit Revaskularisierung von besonderer Bedeutung.

Der in - Abb. 10 abgebildete Zylinder wurde im Bereich einer Osteochondrosis dissecans entnommen, der obere Zylinder mit intakter Knorpelknochenstruktur stellt das Transplantat dar. Die Veränderungen des Gewebes lassen sich bereits makroskopisch gut erkennen (• Abb. 10 a). Typisch ist die vermehrte Sklerosierung der Knochenstruktur bis hin zum Untergang des Gewebes mit Verlust der Kongruenz der Gelenkoberfläche. Auch die Struktur unterhalb des Dissekats ist deutlich verändert.

Um einen ungestörten Gleitvorgang der Gelenkflächen mit regelrechter Duckverteilung gewährleisten zu können, ist ein Resurfacing durch Aufbau der zerstörten Knochenstruktur erforderlich (• Abb. 11).

\section{Osteochondrosis dissecans des Talus}

Sie stellt die dritthäufigste Lokalisation nach Knie und Ellenbogen dar [4]. Wegen der hohen Druckbelastung des Sprunggelenks wird diese Veränderung im Verlauf apparent, stellt aber oft nach Bagatellverletzungen eine Zufallsdiagnose dar.

Problematisch ist sie gelegentlich bei der gutachterlichen Zusammenhangsbeurteilung nach Supinationstraumen, wobei der Kausalzusammenhang dezidiert entschlüsselt werden muss. Nur ein geeigneter Unfallhergang mit entsprechender Krafteinwirkung ist als Ursache anzunehmen [15].

Die in - Abb. 12 a-d dargestellte Osteochondrosis dissecans des Talus wurde von 


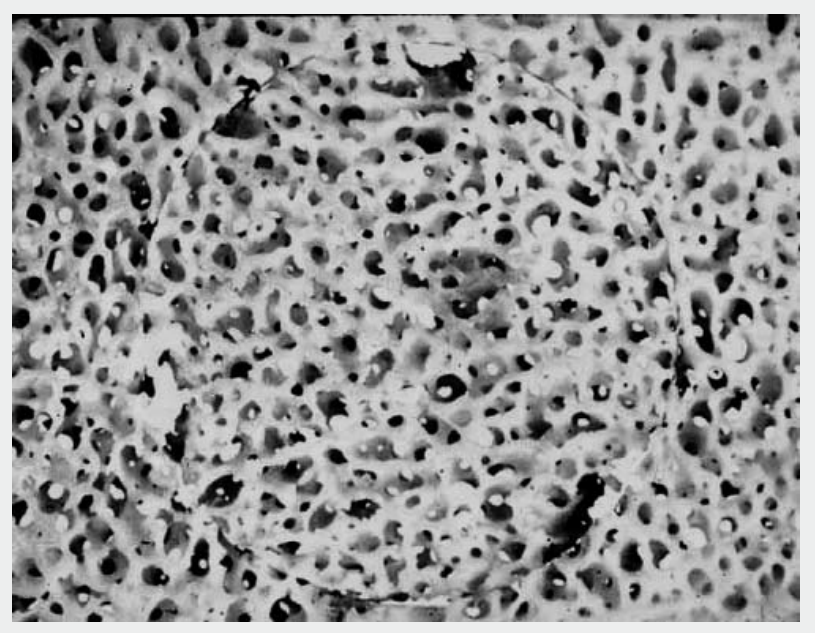

Abb. $7<$ Eingepasste

Transplantatzylinder
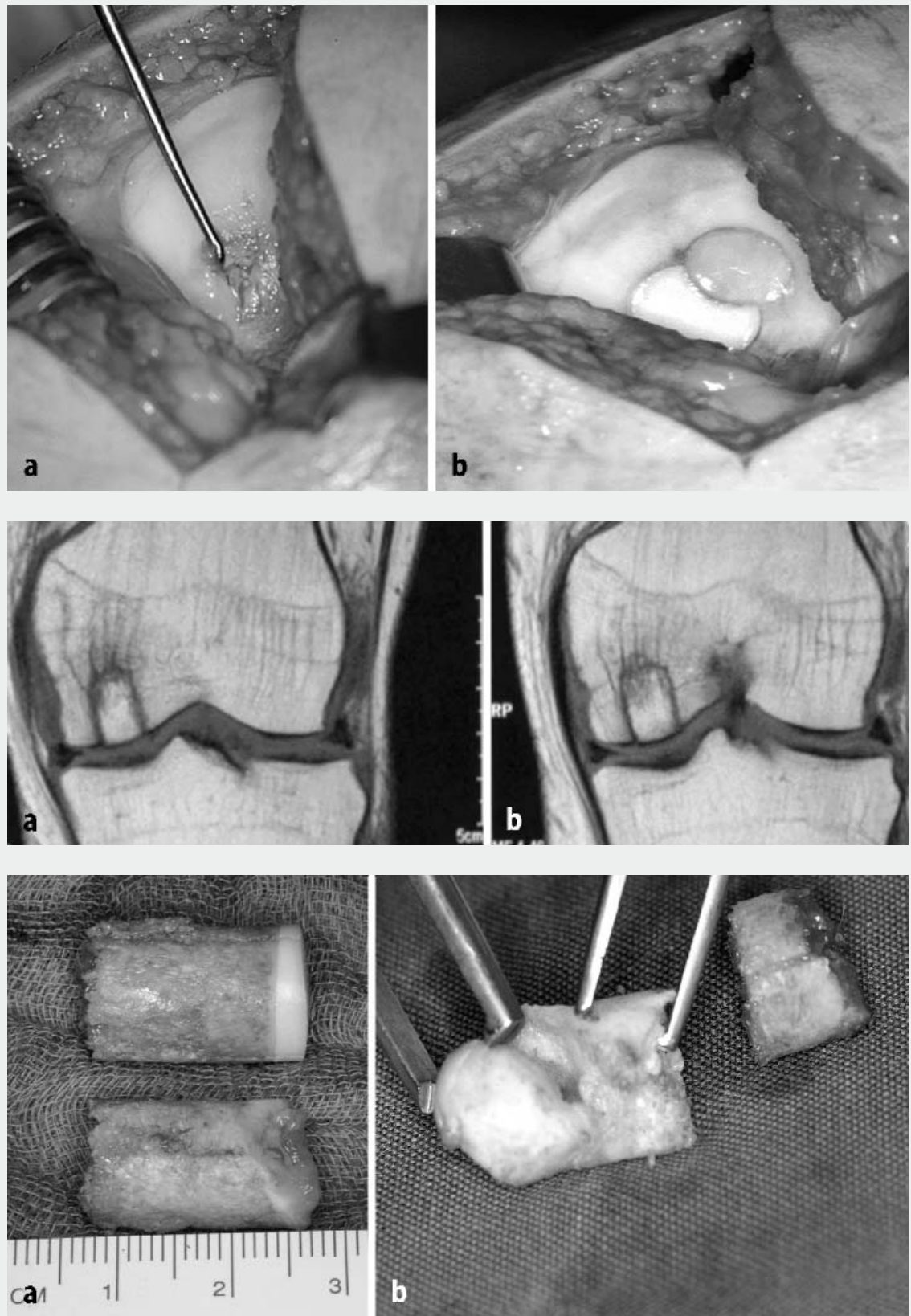

Abb. $8 a, b<$ Defekt des medialen Femurkondylus 1 Jahr nach penetrierender Kniegelenkverletzung durch einen Bolzenschuss (a), Versorgung mit 2 Zylindern (b)

Abb. 9a, b $<$ Mit 2 Zylindern versorgter Kniegelenkdefekt, Magnetresonanztomographie 6 Wochen postoperativ
Abb. 10a, b $<$ Zylinderentnahme bei Osteochondrosis dissecans des Kniegelenks 


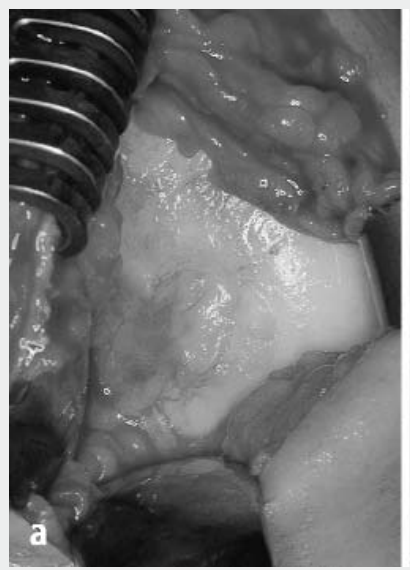

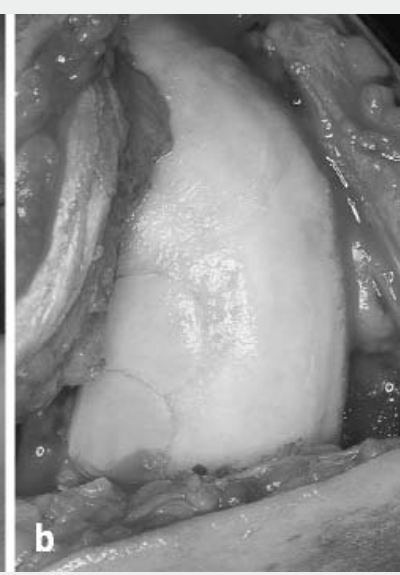

Abb. 11a, b $<$ Tiefe 0steochondrosis dissecans (a), Resurfacing mit 2 Zylindern (b) einer ausgeprägten Beschwerdesymptomatik mit Einschränkung der maximalen Gehstrecke auf unter $1 \mathrm{~km}$ begleitet. Die Magnetresonanztomographie zeigte die Nekrose des betroffenen Areals. Der Defekt konnte mit einem Zylinder rekonstruiert werden. Die MRT-Kontrolle am 5. postoperativen Tag ( $\bullet$ Abb. 12 e,f) lässt wie die Röntgenkontrolle eine anatomische Rekonstruktion der Gelenkstrukturen erkennen. Das nur ausgesprochen geringe Ödem des Marks lässt auf die wenig traumatisierende Technik der Diamantschleifen schließen.

\section{Herkömmliche Methoden}

Bereits vor Einführung der ACT sowie der SDI-Technik wurden Bemühungen unternommen, Defekte des Gelenkknorpels zu behandeln. Ziel waren die Erschaffung oder Transplantation von Gewebe, welches den Belastungsansprüchen des Gelenks gerecht werden kann [9]. Die einzelnen Techniken werden hier nicht detailliert ausgeführt, da die ACT sowie SDITechnik diesen Verfahren nach eingehenden Untersuchungen mit über 10-jährigem Erfahrungszeitraum überlegen sind.

Bekannt ist, dass eine Läsion des Knochengewebes eine unspezifische Reaktion auslöst, wodurch ein Regenerat hervorgebracht wird, welches sich in seiner feingeweblichen Struktur deutlich von hyalinem Knorpel unterscheidet. In der Regel ist die Ausbildung von Narbengewebe zu beobachten. Im günstigsten Fall entsteht ein Faserknorpel, welcher in seiner Qualität vergleichsweise als minderwertig $\mathrm{zu}$ bezeichnen ist [7]. Auf dieser unspezifischen Reaktion begründen sich verschiedenartige Operationsverfahren:

\section{Mikrofrakturierung und Abrasionsplastik}

Durch die oberflächliche Frakturierung oder Shaving mit Läsion der Knochengrenzlamelle werden Mikroblutungen mit nachfolgender Zellmigration von Fibroblasten erreicht, wodurch sich eine Narbenplatte ausbildet.

\section{Mosaikplastik}

Die Entnahme von Knorpel-Knochen-Zylindern erfolgt durch eine Stanze oder eine konventionelle Fräse. Der Substanzdefekt ist in der Regel größer als bei der Entnahmetechnik mittels Diamantfräsen. Eine Pressfit-Technik ist hierbei nicht möglich [5,7]. Die verbleibenden Räume zwischen den einzelnen Zylindern werden durch narbiges Gewebe bzw. Faserknorpel überwuchert.

\section{Biotechnologischer Ausblick}

Die Weiterentwicklung der Methode beinhaltet einerseits die Veränderung der Medien, in denen die Zellkulturen angelegt werden, andererseits die Applikationsformen der Zellkulturen im operativen Vorgehen. Hierdurch sollen die Sicherheit und die einfache Handhabung der Anwendung optimiert werden.

Bisher wurden die Zellen in Patientenoder Kälberserum behandelt und kulti- viert. Mittlerweile stehen auch serumfreie Medien zur Verfügung. Somit wird die Infektionsgefahr durch Viren oder Prionen durch die Kulturmedien ausgeschlossen. Das Teilungsverhalten und die Qualität der Zellen scheinen von diesem Verfahren zu profitieren. Endgültige Erkenntnisse stehen jedoch noch aus.

Die Kopplung der Chondrozyten mit einer Matrix, welche mit den durch den Vermehrungsprozess gewonnenen Zellen beimpft wird, ist ebenfalls möglich. Diese Matrix besteht z. B. aus einer hochgereinigten Kollagenstruktur, welche sowohl eine glatte, zellokklusive Seite als auch eine poröse Membranseite aufweist, die eine Zellbeimpfung und Zellmigration zulässt. Die Matrix besitzt keine Antigenität, da die Telopeptide im Herstellungsprozess abgespalten werden.

Als weitere dreidimensionale Zellträger wurden neben Kollagenen auch Hyaluronat, Polymere, Hydrogele und andere Substanzen verwendet. Diese Grundstoffe sollen einerseits das Handling optimieren, andererseits den Chondrozyten eine optimale Umgebung bieten, um hyalinen Knorpel bilden zu können [12]. Bisher steht der Nachweis aus, dass derartige Zellträger einen Vorteil gegenüber der Zellsuspension erbringen.

Operationstechnisch wäre ein arthroskopisches Verfahren erstrebenswert, welches neben einem problemlosen Handling eine sichere Fixierung der Chondrozyten im Defekt ermöglicht [6]. Da sich eine entsprechende Verfahrensweise noch nicht im klinischen Alltag etablieren konnte, ist die Arthrotomie zur Versorgung von Knorpeldefekten mittels ACT weiterhin Standard.

Bisher bestehen keine Erkenntnisse über das genaue Verhalten der Zellen nach Transplantation. Erkenntnisse hinsichtlich einer Aktivierung oder Bildung von Mediatoren sind nicht vorhanden. Die erschaffene bioaktive Kammer mit implantierten Chondrozyten oder auch das eingebrachte $3 \mathrm{D}$-Vlies stellen somit eine Black Box dar. Ob die lokale oder systemische Unterstützung durch Pharmaka die Vorgänge nach Transplantation begünstigen kann, ist ebenfalls ungeklärt.

Die Verwendung von Stammzellen in der Medizin ist einer angeregten Diskussion unterworfen. Vielfältige Spekulatio- 

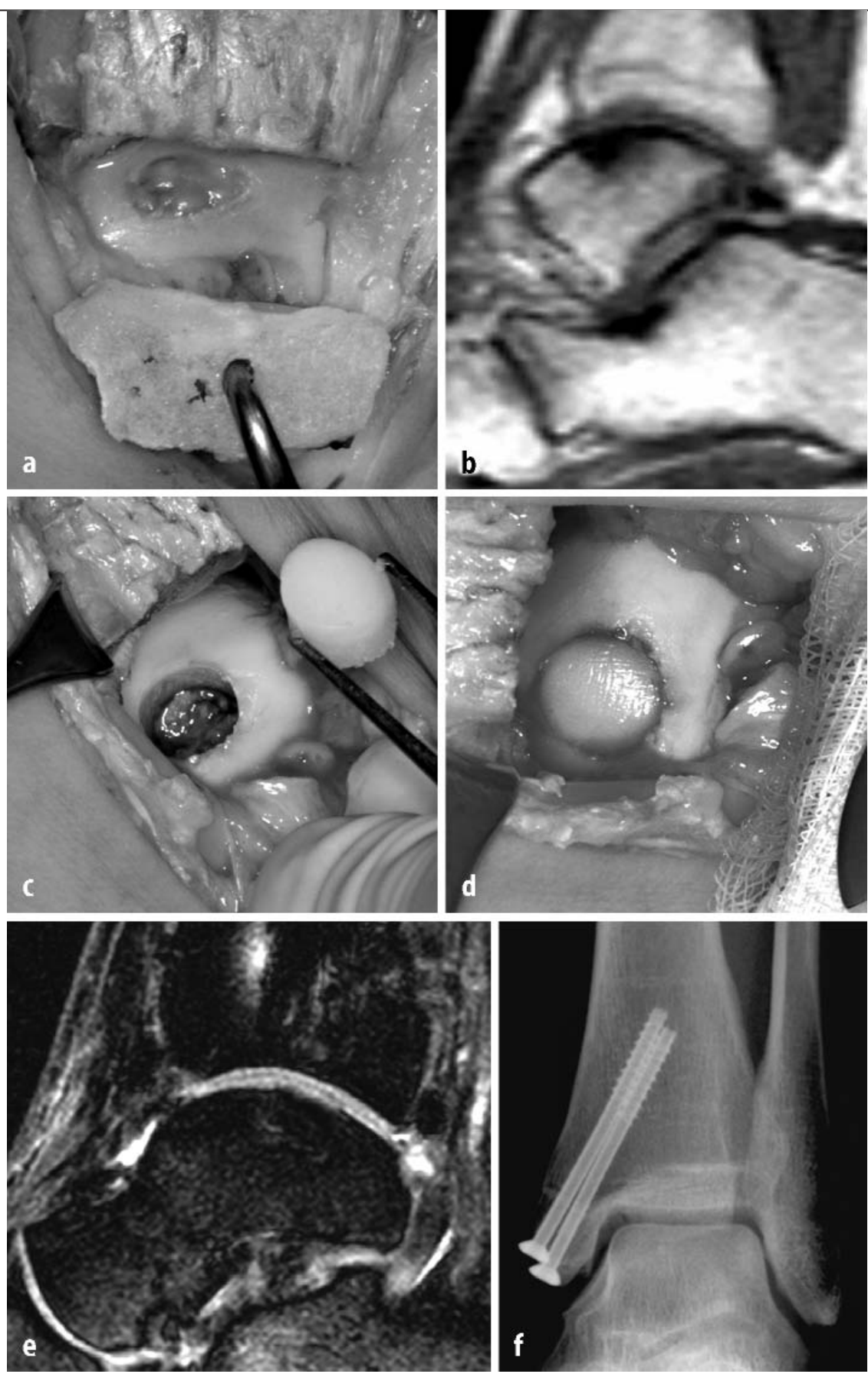

Abb. 12a-f $\Delta$ Osteochondrosis dissecans des Talus, Versorgung mit 1 Zylinder (a-d), MRT-Kontrolle 5 Tage postoperativ $(e, f)$

nen über embryonale und adulte Zellen mit omni- oder pluripotentem Potenzial beflügeln die Visionen, sind aber bisher nicht Bestandteil klinisch etablierter Therapieformen. Durch das kontinuierlich zunehmende Verständnis der genetischen Grundlagen und die Möglichkeit der Steuerung verschiedener Abläufe von Zellak- tivitäten stellt das Tissue engineering aber einen Weg dar, der in Zukunft die Therapieformen maßgeblich verändern kann.

\section{Versorgung von Knorpelschäden durch spezialisierte Zentren}

Die Ersatztechniken, welche im Hauptbelastungsbereich großer Gelenke originären hyalinen oder hyalinähnlichen Ge- lenkknorpel schaffen sollen, haben in den letzten Jahren auf Zellebene bis hin zur Transplantationstechnik deutliche Verbesserungen erfahren. Die Ergebnisse der diesbezüglich durchgeführten Studien können ein optimiertes Outcome belegen.

Der hohe Schwierigkeitsgrad einer Versorgung von komplexen Gelenkschäden mit Kenntnis der biologischen und mechanischen Besonderheiten veranlasst zu dem Entschluss, derartige Verfahren Zentren vorzubehalten. Hier können die finanziell aufwändigen Instrumentarien auch unter wirtschaftlichen Gesichtspunkten sinnvoll eingesetzt werden, wie sie bei der SDI-Technik erforderlich sind.

Erreicht der Versicherte die Sprechstunde eines Zentrums, wurden häufig bereits mehrfach operative Sanierungsversuche vorgenommen, welche aber nicht zum gewünschten Erfolg führten. Diese Vorbedingungen erleichtern die weitere Therapie keinesfalls und können des Weiteren das Outcome der vorgestellten Operationsverfahren negativ beeinträchtigen. Von entscheidender Bedeutung ist es, das geeignete Verfahren zum richtigen Zeitpunkt einzusetzen. Diese Entscheidung kann jedoch lediglich durch einen entsprechend erfahrenen Therapeuten getroffen werden.

Auch müssen Komplikationen letztendlich beherrscht werden und Alternativverfahren im Einzelfall angeboten werden können. Die Indikation der angestrebten Operation sollte nicht darin begründet sein, dass eine Klinik lediglich ein Verfahren beherrscht, wirtschaftlich vertreten kann und somit bevorzugt.

\section{Fazit}

Als Leitlinie der Indikationsstellung verwenden wir die folgende Einteilung:

Primär wird die Unterscheidung zwischen chondraler und osteochondraler Läsion getroffen (•Tabelle 1). Isolierte Knorpeldefekte sind in ihrer Größe, Tiefe und Lokalisation zu bestimmen. Oberflächliche Läsionen können z. B. mittels Abrasionsplastik behandelt werden.

Ist ein drittgradiger Defekt in der Hauptbelastungszone zu finden und überschreitet er $2 \mathrm{~cm}^{2}$, lässt eine ACT ein sehr gutes bis gutes Ergebnis erwarten. Der tibiale Gegenpart 
Tabelle 1

Einteilung der Knorpeldefekte

\begin{tabular}{|lllll}
\hline Grad & I & II & III & IV \\
Chondrale Läsion & Lavage & Lavage, ggf.Débridement & $\begin{array}{l}\text { Lavage, Débridement, } \\
\text { Abrasion/MF, ggf. ACT }\end{array}$ & $\begin{array}{l}\text { Lavage, Débridement, } \\
\text { ACT }\end{array}$ \\
\hline Osteochondrale Läsion & $\begin{array}{l}\text { Vorgehen gemäß } \\
\text { subchondraler Läsion }\end{array}$ & $\begin{array}{l}\text { Vorgehen gemäß } \\
\text { subchondraler Läsion }\end{array}$ & OCT & OCT \\
\hline
\end{tabular}

muss hierbei eine weitgehend unauffällige Struktur aufweisen. Selbiges trifft auf einen viertgradigen Defekt zu.

Bei gravierenden Veränderungen der Knochenstruktur ist eine entsprechende Rekonstruktion anzustreben. Die Art und Ausprägung der ossären Veränderung bestimmen hier das Vorgehen. Inkongruenzen sollten in der tragenden Zone ausgeglichen werden, auch wenn die Oberfläche noch nicht bis auf die Grundplatte geschädigt ist. Bei der begleitenden Ernährungsstörung des Knorpels lassen sich meist tiefer greifende Schäden mit Teilablösungen des Knorpels mit dem Tasthaken feststellen, als die primäre Inspektion vermuten ließ. Inkongruenzen im Hauptbelastungsbereich führen zu einer raschen Degeneration der gegenüberliegenden Gleitfläche. Längerfristiges Zuwarten kann zu Degenerationen führen, welche die Rekonstruktionsmöglichkeiten im weiteren Verlauf unmöglich machen. Bei Schäden Grad III-IV ist die Rekonstruktion der Knorpel-KnochenEinheit zeitnah anzustreben.

Letztendlich handelt es sich aber immer um eine individuelle Entscheidung, welche mit dem Patienten im Einzelfall getroffen werden muss.

\section{Korrespondierender Autor Dr. R. Feil}

Klinik für Unfall- und Wiederherstellungschirurgie, Unfallkrankenhaus Berlin, Warener Straße 7, 12683 Berlin,

E-mail:Roman.Feil@ukb.de

Interessenkonflikt: Keine Angaben

\section{Literatur}

1. Bentley G, Biant LC, Carrington RW et al. (2003) A prospective, randomised comparison of autologous chondrocyte implantation versus mosaicplasty for os teochondral defects in the knee.J Bone Joint Surg Br 85: 223-230

2. Bobic V (1999) Die Verwendung von autologen Knochen-Knorpel-Transplantaten in der Behandlung von Gelenkknorpelläsionen. Orthopäde 28: 19-25
3. Brittberg M, Lindahl A, Nilsson A et al. (1995) Treatment of deep cartilage defects in the knee with autologous chondrocyte transplantation. N Engl J Med 331: 889895

4. Bruns J, Behrens P (1998) Osteochondrosis dissecans, Ätiologie und Pathophysiologie. Arthroskopie 11: 166-176

5. Deaenert K, Draenert Y (1988) A new procedure for bone biopsies and cartilage and bone transplantation. Sandorama III/IV: 33-40

6. Ergelett Ch, Sittinger M, Lahm A (2003) The arthroscopic implantation of autologous chondrocytes for the treatment of full-thickness cartilage defects of the knee joint. Arthroscopy 19: 108-110

7. Hochstein P, Glatzel U, Schmickal T et al. (2002) Klinische Erfahrungen mit der Knorpel-Knochen-Transplantation. Trauma Berufskrankh [Suppl 2] 4: S110-114

8. Horas U, Schnettler R, Pelinkovic D et al. (2000) Knorpelknochentransplantation versus autogene Chondrocytentransplantation. Chirurg 71: 1090-1097

9. Imhoff AB, Öttel G (1998) Osteochondrale AutograftTransplantation in verschiedenen Gelenken. In: Imhoff AB, Burghart A (Hrsg) Knieinstabilität - Knorpelschaden.Steinkopff, Darmstadt

10. Insall JN, Joseph DM, Msika C (1984) High tibial osteotomie for varus gonarthrosis. A long-term follow-upstudy. J Bone Joint Surg Am 66: 1040-1048

11. Laprell H,Petersen W (2001) Autologous osteochondra transplantation using the diamond bone-cutting system: $6-12$ years' follow-up of 35 patients with ostechondral defects at the knee joint. Orthop Trauma Surg 121: 248-253

12. Makarand V, Sittinger M (2002) Tissue engineering: advances in in vitro cartilage generation. Trends Biotechnol 20: 8

13. Martin JA, BruckwalterJA (1996) Articular cartilage aging and degeneration. Sports Med Arthrosc 4: 263-275

14. Peterson L, Brittberg M, Kiviranta I et al. (1998) Autologous chondrocyte transplantation: 2-10 year followup in 219 patients. Am Acad Orthop Surg 74

15. Schönberger A, Merthens G, Valentin H (2003) Arbeitsunfall und Berufskrankheit, 7. Aufl. Schmidt, Berlin, S 714-716

16. Steinwachs M, Ergelett Ch, Lahm A et al. (1999) Klinische und zellbiologische Aspekte der autologen Chondrocytenimplantation. Unfallchirurg 102: 855-860 